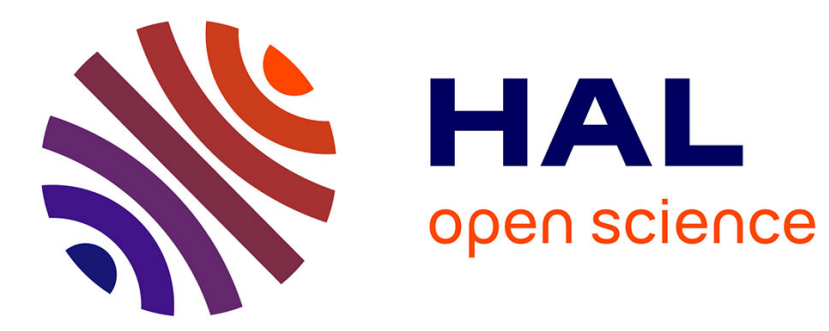

\title{
Large scale kronecker product on supercomputers
}

Claude Tadonki

\section{To cite this version:}

Claude Tadonki. Large scale kronecker product on supercomputers. 23rd International Symposium on Computer Architecture and High Performance Computing - SBAC-PAD 2011 - WAMCA 2011, Oct 2011, Victoria, Brazil. pp.1-4, 10.1109/WAMCA.2011.10 . in2p3-00702588

\section{HAL Id: in2p3-00702588 https://hal.in2p3.fr/in2p3-00702588}

Submitted on 30 May 2012

HAL is a multi-disciplinary open access archive for the deposit and dissemination of scientific research documents, whether they are published or not. The documents may come from teaching and research institutions in France or abroad, or from public or private research centers.
L'archive ouverte pluridisciplinaire HAL, est destinée au dépôt et à la diffusion de documents scientifiques de niveau recherche, publiés ou non, émanant des établissements d'enseignement et de recherche français ou étrangers, des laboratoires publics ou privés. 


\title{
Large scale kronecker product on supercomputers*
}

\author{
Claude Tadonki \\ MINES ParisTech - CRI (Centre de Recherche en Informatique) - Mathematiques et Systèmes \\ 35 , rue Saint-Honoré \\ 77305, Fontainebleau-Cedex \\ Laboratoire de l'Accélérateur Linéaire/IN2P3/CNRS \\ University of Orsay, Faculty of Sciences, Bat. 200 \\ 91898 Orsay Cedex (France) \\ claude.tadonki@u-psud.fr
}

\begin{abstract}
The Kronecker product, also called tensor product, is a fundamental matrix algebra operation, which is widely used as a natural formalism to express a convolution of many interactions or representations. Given a set of matrices, we need to multiply their Kronecker product by a vector. This operation is a critical kernel for iterative algorithms, thus needs to be computed efficiently. In a previous work, we have proposed a cost optimal parallel algorithm for the problem, both in terms of floating point computation time and interprocessor communication steps. However, the lower bound of data transfers can only be achieved if we really consider (local) logarithmic broadcasts. In practice, we consider a communication loop instead. Thus, it becomes important to care about the real cost of each broadcast. As this local broadcast is performed simultaneously by each processor, the situation is getting worse on a large number of processors (supercomputers). We address the problem in this paper in two points. In one hand, we propose a way to build a virtual topology which has the lowest gap to the theoretical lower bound. In the other hand, we consider a hybrid implementation, which has the advantage of reducing the number of communicating nodes. We illustrate our work with some benchmarks on a large SMP 8-Core supercomputer.
\end{abstract}

\section{Introduction}

The Kronecker product is a basic matrix algebra operation, which is mainly used for multidimensional modeling in number of specialized fields[5]: Stochastic Automata

\footnotetext{
*This work was partly supported by the PetaQCD (ANR) project.
}

Networks (SAN) [2, 3, 4], Fast Fourier Transform (FFT), Fast Poisson Solver (FPS) [9, 10], Quantum Computation (QC) [6] and Lattice Quantum Chromodynamics [8]. Considering a principal matrix expressed as a Kronecker product of several matrices, iterative schemes require to repeatedly multiply such a matrix by a vector. Formally, we are given $N$ square matrices $A^{(i)}$ of sizes $n_{i}, i=1, \cdots, N$, and a vector $x$ of length $L=n_{1} n_{2} \cdots n_{N}$, and we need to compute $y$ (of length $L$ ) given by

$$
y=x\left(\bigotimes_{i=1}^{N} A^{(i)}\right)
$$

It is well-known that we should not compute the matrix explicitly before performing the multiplication, as this would require a huge memory to store that matrix and will yield redundant computations. A cost optimal algorithm for this computation proceeds in a recursive way, consuming one matrix $A^{(i)}$ after another [7]. Consequently, traditional parallel routines for matrix-vector product cannot be considered. When starting with the recursive algorithm as a basis, any parallel scheme will involve a set of data communication at the end of each iteration. The cost of this communication is the main challenge for this problem, especially with a large number of processors, because there is a significant interleave between the (probably virtual) communication links. Moreover, in order to reduce the cache misses due to an increasing stride from one iteration to the next one, array reshuffling is sometimes considered, and this complicates the communication topology.

In [7], we have proposed an efficient parallel algorithm which achieves the multiplication without explicit shuffling and requires a minimal number of communication steps. However, the real cost of each communication step depends on the virtual topology and the way the transfers are really 
performed. This problem was left open in the paper because of the modest size of the parallel computers considered (up to 256 processors). In this paper, we provide an algorithm to construct an efficient topology, in addition to a hybrid implementation using OpenMP[11] on the computing multicore nodes. With this contribution, we keep the global efficiency of the original algorithm on a larger number of processors as illustrated by some benchmark results. The rest of the paper is organized as follows. Section 2 gives an overview of the original algorithm. This is followed in section 3 by a discussion on its complexity and the position of the problem. We describe our heuristic to find an efficient topology in section 4 . We discuss the hybrid implementation and section 5. In section 6, we display and comment our benchmark results. Section 7 concludes the paper.

\section{Original parallel algorithm}

We restate our parallel algorithm in order to provide a self-contained material, the reader could refer to [7] for more details. From (1) and using the so-called canonical factorization, we obtain the recursive scheme defined by (2)

$$
\left\{\begin{array}{l}
V^{(N+1)}=x \\
V^{(s)}=V^{(s+1)}\left(I_{n_{1} \cdots n_{s-1}} \otimes A^{(s)} \otimes I_{n_{s+1} \cdots n_{N}}\right)
\end{array}\right.
$$

which leads at the last step to $V^{(1)}=x \otimes_{i=1}^{N} A^{(i)}$. Our parallelization of the recursive computation expressed by equation (2) can be defined as follows. Given $p$ processors (assuming that $p$ divides $L=n_{1} n_{2} \ldots n_{N}$ ), we proceed as follows. We first compute a sequence of $N$ integers $p_{i}$ such that $p=p_{1} p_{2} \ldots p_{N}$ and $p_{i}$ divides $n_{i}, i=$ $1,2, \ldots, N$. Considering a multidimensional indexation, we say that each processor $\left(a_{1}, a_{2}, \cdots, a_{N}\right)$ computes the entries $\left(b_{1}, b_{2}, \cdots, b_{N}\right)$ of $V^{(s)}$ such that $b_{i} \bmod p_{i}=a_{i}$, $i=1,2, \cdots, N$. A complete description of the parallel algorithm is given by $\mathrm{Alg}$. 1 . Note that the send and receive occurrences can be combined into a single sendreceive call because of the symmetry of the topology.

\section{Communication complexity}

Our scheduling onto $p$ processors is based on a decomposition $\left(p_{1}, p_{2}, \cdots, p_{N}\right)$ such that $p_{i}$ divides $n_{i}$, and $p_{1} p_{2} \cdots p_{N}=p$. In theory, algorithm Alg. 1 performs $\log (p)$ parallel communication steps when executed with $p$ processors. Indeed, one local broadcast occurs at the end of each step $s$, thus we do $\log \left(p_{1}\right)+\log \left(p_{2}\right)+\cdots+\log \left(p_{N}\right)=$ $\log \left(p_{1} p_{2} \cdots p_{N}\right)=\log (p)$ parallel communication steps. This assumes that, at a given step $i$, we perform $\log \left(p_{i}\right)$ parallel transfers (local broadcast to $p_{i}$ processors by each processor). However, in practice, we issue $p_{i}-1$ transfers (communication loop). Thus, the gap between $p_{i}-1$ and $\log \left(p_{i}\right)$ becomes important for larger $p_{i}$. Actually, each processor performs $p_{1}+p_{2}+\cdots+p_{N}$ transfers in total. On a larger cluster, there will be an additional overhead coming from the gap between the virtual topology and the physical topology. We first focus on how to find a decomposition which reduces the measure $p_{1}+p_{2}+\cdots+p_{N}$.

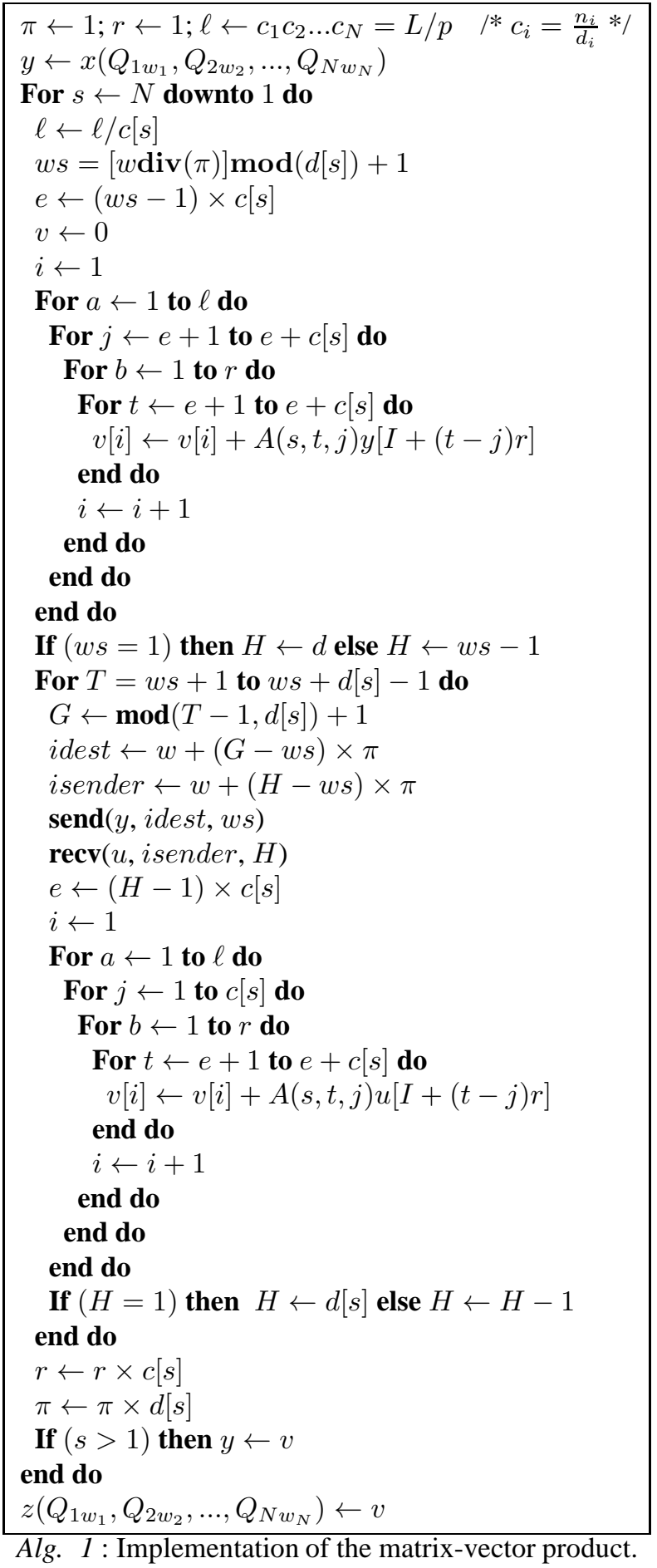




\section{Heuristic for an efficient topology}

We propose the algorithm Alg. 2 to find an efficient decomposition for a given number of processors $p$, which is a factor of $n_{1} n_{2} \cdots n_{N}$.

\begin{tabular}{|l|}
\hline$d \leftarrow p$ \\
$\{$ Starting decomposition $\}$ \\
For $i \leftarrow 1$ to $N$ do \\
$p_{i} \leftarrow \operatorname{gcd}\left(d, n_{i}\right)$ \\
$d \leftarrow \frac{d}{p_{i}}$ \\
enddo \\
$\{$ Recursive refinement $\}$ \\
For $i \leftarrow 1$ to $N$ do \\
For $j \leftarrow 1$ to $N$ do \\
$\quad \alpha \leftarrow \operatorname{gcd}\left(p_{i}, \frac{n_{j}}{p_{j}}\right)$ \\
if $\left((\alpha>1) \wedge\left(p_{i}>c p_{j}\right)\right)$ \\
$\quad p_{i} \leftarrow \frac{d_{i}}{\alpha}$ \\
$\quad p_{j} \leftarrow \alpha d_{j}$ \\
endif \\
enddo \\
enddo \\
Alg. $2:$ Heuristic for an efficient decomposition
\end{tabular}

The principle of Alg. 2 is the following. We start with a gcd decomposition. Next, we refine it using the fact that if $p_{i}>\alpha p_{j}$, with $\alpha$ a non trivial factor of $p_{i}$, then $p_{i} / \alpha+$ $\alpha p_{j}<p_{i}+p_{j}$. It is thus rewarding to replace $p_{i}$ (resp. $p_{j}$ ) by $p_{i} / \alpha$ (resp. $\alpha p_{j}$ ). Once this is done, it is clear that on a larger cluster (i.e. large value of $p$ ), all these simultaneous transfers will exacerbate the communication overhead and certainly slowdown the global performance. Fortunately, most modern supercomputers are built up with multicore nodes. Thus, a hybrid implementation, which combines the standard distributed memory implementation with a shared memory program (SMP) on the nodes, will overcome the problem by reducing the number of communicating nodes.

\section{SMP implementation}

We chose to use OpenMP to derive our shared memory code. Looking at Alg. 1, we decide to put the loop distribution pragma over the $a$ loop. In order to do so, we first need to remove the $i \leftarrow i+1$ incrementation and directly calculate the $i$ index, which is given by $i \leftarrow c[s] \times r \times(a-1)+r \times(j-1)+b$. Now, the length $\ell$ where the loop blocking will occur varies with $s$ $(\ell \leftarrow \ell / c[s])$. Thus, we need to keep it being a factor of the (fixed) number of threads. We achieve it by splitting the main loop into two parts, means isolating the case $s=N$ and then enclose the rest $(s=N-1, N-2, \cdots, 1)$ into a parallel section. Moreover, since the number of nodes is now reduced to $p / T$ ( $T$ is the number of OpenMP threads), we need to adapt our primarily decomposition such that $\ell$ remains a factor of $T$. The general way to do that is to split the loop over $s$ at the right place (not only the extremal iteration), but this would be better implemented with Posix threads library, because we could dynamically manage the threads to handle desired loop partitioning (this is left for future work). We now show the impact of our strategy on benchmark results. Interested reader can download the source code at

http://www.omegacomputer.com/staff/tadonki/codes/kronecker.f

\section{Experimental results}

We consider a SMP 8-core cluster named JADE [12]. The whole cluster JADE is composed of 1536 compute nodes (i.e. $1536 \times 8=12288$ cores of Harpertown type processors) and 1344 compute nodes of nehalem type processor (1344 x $8=10752$ cores). The network fabric is an Infiniband (IB 4x DDR) double planes network for the first part of the machine ( Harpertown), whereas 4 drivers InfiniBand 4X QDR provide 72 ports IB 4X QDR on output of each IRU of the second part of the machine $(576 \mathrm{Go} / \mathrm{s})$.

We choose $N=6$ square matrices of orders $20,36,32$, 18,24 , and 16, which means a principal matrix of order $L=159252480$. We first show in table 1 the results of the pure MPI code. The decomposition obtained with our algorithm is marked with a star and is surrounded by two alternative decompositions (the one obtained by a basic ged decomposition and the less distributed one) to illustrate the difference.

\begin{tabular}{|r|c|c|}
\hline $\mathrm{p}$ & decomposition & time $(\mathrm{s})$ \\
\hline 32 & $(4,1,8,1,1,1)$ & $2.06 \mathrm{~s}$ \\
\hline 32 & $(2,2,2,2,2,1)^{*}$ & $1.62 \mathrm{~s}$ \\
\hline 32 & $(1,1,32,1,1,1)$ & $4.14 \mathrm{~s}$ \\
\hline \hline 180 & $(20,9,1,1,1,1)$ & $0.75 \mathrm{~s}$ \\
\hline 180 & $(5,3,2,2,3,1)^{*}$ & $0.34 \mathrm{~s}$ \\
\hline 180 & $(10,6,1,1,3,1)$ & $0.49 \mathrm{~s}$ \\
\hline \hline 720 & $(20,36,1,1,1,1)$ & $1.20 \mathrm{~s}$ \\
\hline 720 & $(10,3,2,2,3,2)^{*}$ & $0.23 \mathrm{~s}$ \\
\hline 720 & $(10,9,4,2,1,1)$ & $0.35 \mathrm{~s}$ \\
\hline \hline 2880 & $(20,36,4,1,1,1)$ & $1.47 \mathrm{~s}$ \\
\hline 2880 & $(10,6,2,2,6,2)^{*}$ & $1.20 \mathrm{~s}$ \\
\hline 2880 & $(20,12,2,2,3,1)$ & $1.32 \mathrm{~s}$ \\
\hline \hline 4320 & $(20,36,2,3,1,1)$ & $1.48 \mathrm{~s}$ \\
\hline 4320 & $(10,3,4,3,3,4)^{*}$ & $0.92 \mathrm{~s}$ \\
\hline 4320 & $(20,18,4,3,1,1)$ & $1.34 \mathrm{~s}$ \\
\hline
\end{tabular}

Table 1. MPI implementation timings

From Table 1, we see that for a given number of processors, the partition obtained with our procedure can im- 
prove the global performance by a factor from 2 to 5 (see $p=720$ ). However, when the number or MPI processes increases, we see that we lose the scalability, because data communication severely dominates (the code is cost optimal for floating point operations). We now see how this is improved using a hybrid implementation. We reconsider the previous best decompositions as baseline and compare each of them with the corresponding hybrid configuration. For each number of cores in $\{4320,2880,720\}$, we consider a $k$-cores SMP clustering, $k \in\{1,4,8\}$.

\begin{tabular}{|r|c|c|r|r|}
\hline \#MPI & decomposition & \#threads & time & speedup \\
\hline 4320 & $(10,3,4,3,3,4)$ & 1 & $0.92 \mathrm{~s}$ & 1 \\
\hline 1080 & $(5,3,2,3,3,4)$ & 4 & $0.16 \mathrm{~s}$ & 5.75 \\
\hline 540 & $(5,3,2,3,3,2)$ & 8 & $0.12 \mathrm{~s}$ & 7.67 \\
\hline \hline 2880 & $(10,6,2,2,6,2)$ & 1 & $1.20 \mathrm{~s}$ & 1 \\
\hline 360 & $(5,3,2,3,3,4)$ & 8 & $0.16 \mathrm{~s}$ & 7.5 \\
\hline \hline 720 & $(10,3,2,3,3,2)$ & 1 & $0.23 \mathrm{~s}$ & 1 \\
\hline 90 & $(5,3,2,1,3,1)$ & 4 & $0.45 \mathrm{~s}$ & 0.51 \\
\hline
\end{tabular}

Table 2. Hybrid (MPI+OpenMP) code timings

We can see from Table 2 that we are close to a linear (threads) speedup with 4320 and 2880 cores. This is due to the fact that the global computation time was really dominated by data communication and synchronization mechanism. For a smaller number of cores, we see that we start loosing the benefit of the SMP implementation. This is due the (predictable) cache misses penalty coming from the stride $(t-j) \times r$ in Alg. 1, which is increasingly bigger since $r$ does. We could use larger number of cores, but we our experimental configuration sufficiently illustrative of what we need to show and how our solution contributes to the issues.

\section{Conclusion}

The problem of multiplying a vector by a Kronecker product of matrices is crucial in stochastic sciences and is a computationally challenging task for large instances. In order to avoid a memory bottleneck and redundant computation, a recursive scheme has been mathematically formulated, for which corresponding efficient implementations are expected. In one hand, the increasing loop stride needs to be handled carefully in order to reduce the impact of caches misses. This aspect really dominates and thus needs to be seriously taken into account in the performance analysis. In the other hand, the parallelization requires an important number of parallel transfers, which could become problematic on large clusters. This paper provides a contribution on both aspects, based on a cost optimal solution (floating point computation point of view) from the literature. Our solution is a combination of a heuristic procedure to build an efficient virtual topology and the use of hybrid programming paradigm. Our experimental results illustrate the improvement of our contribution, and evidence the need of a compromise on large clusters.

\section{References}

[1] M. Davio, Kronecker Products and Shuffle Algebra, IEEE Trans. Comput., Vol. C-30, No. 2, pp. 116-125, 1981.

[2] P. Fernandes, B. Plateau, and W. J. Stewart, Efficient Descriptor-Vector Multiplications in Stochastic Automata Networks, INRIA internal report No. 2935 , July 1996.

[3] Benoit, Anne and Fernandes, Paulo and Plateau, Brigitte and Stewart, William J., On the benefits of using functional transitions and Kronecker algebra, Performance Evaluation, Vol. 58(4), pp. 367-390, 2000 .

[4] Benoit, Anne and Plateau, Brigitte and Stewart, William J., Memory-efficient Kronecker algorithms with applications to the modeling of parallel systems, Future Gener. Comput. Syst., Vol. 22(7), pp. 838$847,2006$.

[5] J. Granta, M. Conner, and R. Tolimieri, Recursive fast algorithms and the role of the tensor product, IEEE Transaction on Signal Processing, 40(12):2921-2930, December 1992.

[6] P. W. Shor, Quantum Computing, Proceeding of the ICM Conference, 1998.

[7] C. Tadonki and B. Philippe, Parallel Multiplication of a Vector by a Kronecker Product of Matrices, Journal of Parallel and Distributed Computing and Practices, Parallel Distributed Computing Practices PDCP, volume 3(3), 2000.

[8] C. Tadonki, G. Grosdidier, and O. Pene, An efficient CELL library for lattice quantum chromodynamics, ACM SIGARCH Computer Architecture News, Vol. 38(4), 2011.

[9] C.Tong and P. N. Swarztrauber, Ordered Fast Fourier Transforms on Massively Parallel Hypercube Multiprocessor, Journal of Parallel and Distributed Computing 12, 50-59, 1991.

[10] C. Van Loan, Computational Framework for the Fast Fourier Transform, SIAM, 1992.

[11] http: / / openmp.org/

[12] http://www.cines.fr 